\title{
Plasma exchange in Goodpasture syndrome associated with Turner's syndrome: A case report
}

\author{
Jiao LP, Fan JF, Sun Q, *Shen Y
}

National Key Discipline of Pediatrics (Capital Medical University)

Ministry of Education, Beijing Children's Hospital, Capital Medical University, Beijing, 100045, China

\begin{abstract}
Background: Good pasture syndrome (GPS) has been paid much attention recently for the dangerous illnessand high mortality.

Objective: To investigate the efficiency of plasma exchange (PE) to treat Goodpasture syndrome (GPS) in children associated with Turner's syndrome.

Method: We report a case of a 15 year old female with GPS and Turner's syndrome. The patient has intermittent fever and cough for 45 days and oliguria for 6 days. Turner's syndrome was determined through blood karyotype analysis, and GPS was diagnosed because the patient was negative for antinuclear antibodies and antineutrophil cytoplasmic antibodies (ANCA), but positive for anti-glomerular basement membrane (anti-GBM) antibodies (200 RU/ml). PE was carried out in combination with immunosuppression therapy.

Results: The results show PE treatment can efficiently decrease the levels of anti-GBM antibodies. The antibody levels were $>200 \mathrm{RU} / \mathrm{ml}$ and $184 \mathrm{RU} / \mathrm{ml}$ before and after the first PE treatment, respectively. The removal efficiency were 40\%, 47\%, $42 \%, 54 \%, 52 \%$ for the fifth, sixth, seventh, eighth and ninth PE procedures, respectively.

Conclusion: The therapy with PE, hemodialysis, pulse methylprednisolone followed by oral prednisone and cyclophosphamide greatly contributed to improvement of this patient's condition, and resolved the patient's pulmonary haemorrhage. All these results demonstrate that PE contributed efficiently to the treatment for GPS in children.
\end{abstract}

Keywords: Goodpasture syndrome (GPS), Turner's syndrome, plasma exchange (PE).

African Health Sciences 2012; (4): 572 - 575 http://dx.doi.org/10.4314/ahs.v12i4.29

\section{Introduction}

Goodpasture syndrome (GPS) is rare in children. The role of anti-glomerular basement membrane (anti-GBM) antibodies in the pathogenesis of GPS is well-known, and plasma exchange (PE) has been used for antibody removal ${ }^{1-2}$. We reported the first case of GPS associated with Turner's syndrome in children. The patient was treated with a combination of PE, steroids and immunosuppression, which has previously been reported to be effective. The levels of anti-GBM antibodies were tested pre- and post$\mathrm{PE}$, and the efficiency of removal was calculated to evaluate the efficiency of PE therapyfor treatment of GPS.
*Corresponding author:
Ying Shen
Department of Nephrology
Beijing Children's hospital
Number 56 Nanlishi Road
Xicheng district
Beijing, China
Tel: +86-10-59616626
E-mail: ving116sh@sohu.com

\section{Case report}

A 15-year-old female was hospitalized in our hospital with a 45-day history of intermittent fever and cough. She received antibiotics from her local hospital, but symptoms did not improve. Oliguria was developed at 6 days before admission, and was accompanied by two episodes of blood-streaked sputum and pallor. Haemodialysis (HD) was performed 2 days before admission. She did not experience any rash or joint swelling.

Her past medical history includes a diagnosis of Turner's syndrome diagnosed at the age of 14 years and 1 month by blood karyotype analysis (figure 1). Her height was $132 \mathrm{~cm}$ at diagnosis and growth hormone $(\mathrm{GH})$ treatment was commenced, but was discontinued by her parents 8 months later (height $140 \mathrm{~cm}$ ) due to an upper respiratory tract infection. There was no family history of renal disease.

This patient was admitted in the first affiliated Hospital of Harbin Medical University on May 2th, 2010. On admission she was in no apparent distress, and weight was $35.5 \mathrm{~kg}$. Eyelid oedema was

African Health Sciences Vol 12 Issue 4 December 2012 
noted. She had normal breath sounds, and heart and abdominal examinations were normal. Cubitus valgus and lower limb oedema were observed, with a good range of motion of all joints. Breasts and pubic hair were Tanner stage 1 . No adenopathy was detected.

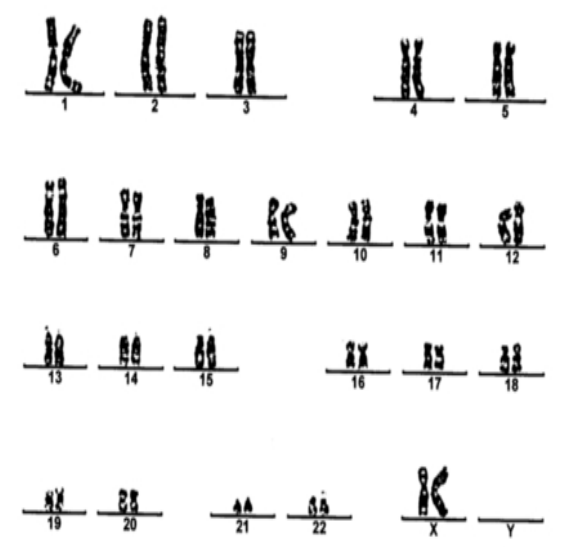

Fig. 1

209x119mm (72 x 72 DPI)

Figure 1: Representative figure of blood karyotype analysis

Blood sampling before HD revealed blood urea nitrogen (BUN) $36.33 \mathrm{mmol} / \mathrm{L}$, serum creatinine (Cr) $974.6 \mu \mathrm{mol} / \mathrm{L}(11.0 \mathrm{mg} / \mathrm{dL})$, normal liver function, haemoglobin $(\mathrm{Hb}) 85 \mathrm{~g} / \mathrm{L}$, white blood cells $11.49 \times 10^{9} / \mathrm{L}$, platelets $193 \times 10^{\circ} / \mathrm{L}$ and Creactive protein $160 \mathrm{mg} / \mathrm{L}$. Urinalysis showed $2+$ protein and $>50$ red blood cells per high-power field. Complement levels (C3, C4) were normal. Antinuclear antibodies and antineutrophil cytoplasmic antibodies (ANCA) were negative. Anti-glomerular basement membrane (anti-GBM) antibody levels were high at $>200 \mathrm{RU} / \mathrm{ml}$ (enzyme-linked immunosorbent assay, normal value $<20 \mathrm{RU} / \mathrm{ml}$ ). Abdominal ultrasound revealed enlarged kidneys with increased cortical echogenicity. Chest computed tomography scan revealed mild bilateral interstitial and parenchymal infiltrates.

Renal biopsy was performed 11 days after admission. Light microscopy of 12 glomeruli revealed 11 glomeruli with global sclerosis and 1 with segmental sclerosis. Immunofluorescence staining for IgG, IgA, IgM, C3 and C1q was negative. A diagnosis was made of sclerosing glomerulonephritis. Pulmonary haemorrhage was diagnosed on flexible bronchofibreoscopy (many red cells and occasional haemosiderin in the irrigating solution, figure 2).

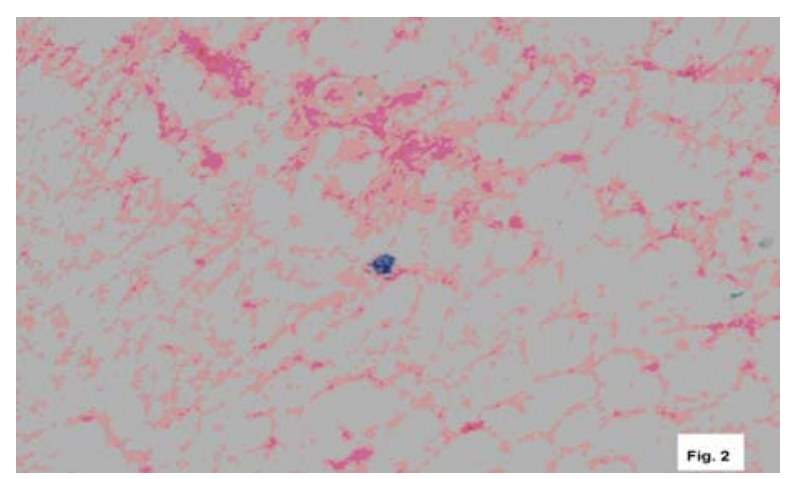

479x361 mm (72 x $72 \mathrm{DPI})$

Figure 2: Irrigating solution collected by flexible bronchofiberscope: (Toluidine blue dyeing $\times 400$ )

HD was continued after admission. Plasma exchange (PE) was performed when GPS was diagnosed on day 11, using a Gambro plasma filter and a PRISMA machine. Intravenous access was via femoral vein cannulation. Volume exchanged was $40 \mathrm{ml} / \mathrm{kg}(1$, $400 \mathrm{ml}$ ) with blood flow $150 \mathrm{ml} / \mathrm{min}$. Fresh frozen plasma $(1,000 \mathrm{ml})$ and substitute plasma $(400 \mathrm{ml})$ were used as replacement fluid, and heparin sodium was used as anticoagulant. The duration of each exchange was 2 hours, with nine exchanges completed. Anti-GBM antibody levels were tested pre- and post-PE for each exchange. Antibody levels were $>200 \mathrm{RU} / \mathrm{ml}$ before and $184 \mathrm{RU} / \mathrm{ml}$ after the first PE. After the fourth PE, antibody levels remained $<200 \mathrm{RU} / \mathrm{ml}$. Removal efficiency was $40 \%$, $47 \%, 42 \%, 54 \%$ and $52 \%$ for the fifth, sixth, seventh, eighth and ninth PE procedures, respectively (table 1).

After PE, HD and pulse methylprednisolone followed by oral prednisone and cyclophosphamide, the patient's pulmonary haemorrhage resolved. Renal function did not improve and she continued to require intermittent $\mathrm{HD}$. 
Table 1: Determination of anti-GBM antibody pre- and post-PE (RU/ml)

\begin{tabular}{|c|c|c|c|c|c|c|c|c|c|}
\hline & First & Second & Third & Fourth & Fifth & Sixth & Seventh & Eighth & Ninth \\
\hline$\overline{\text { Pre-PE }}$ & $>200$ & $>200$ & 185 & $>200$ & 146 & 135 & 113 & 68 & 63 \\
\hline Post-PE & 184 & 139 & 120 & 118 & 88 & 71 & 65 & 31 & 30 \\
\hline $\begin{array}{l}\text { Removal } \\
\text { efficiency }\end{array}$ & - & - & 35 & - & 40 & 47 & 42 & 54 & 52 \\
\hline
\end{tabular}

\section{Discussion}

GPS is characterized by rapidly progressive glomerulonephritis, pulmonary haemorrhage and the presence of anti-GBM antibodies. Anti-GBM disease accounts for approximately $10-20 \%$ of patients with rapidly progressive glomerulonephritis ${ }^{3}$. GPS is rare in children, and usually occurs from ages $6-13 .{ }^{4}$, although the youngest reported case was 11 months old ${ }^{5}$. Onestudy of GPS in children reported a male to female ratio of 0.43 , with positive anti-GBM antibody levels in all patients who were tested ${ }^{4}$. Our patient was a female adolescent, with an anti-GBM antibody level above the upper limit of the test range and negative ANCA. Microscopy 17 days after the onset of oliguria showed global sclerosis in $92 \%$ of glomeruli and segmental sclerosis in $8 \%$, indicating rapid progression. She had mild symptoms of hemorrhage and thus a flex bronch was performed to confirm the diagnosis.

PE was first used for the removal of immune complexes in GPS in the early 1970s, and is now a grade $1 \mathrm{~B}$ recommendation ${ }^{6}$. In the current case, serum anti-GBM antibody levels decreased following PE, confirming that PE is an effective therapeutic approach for GPS in children. Efficiency of antibody removal was $35-54 \%$, which is similar to that demonstrated by double-filtration plasmapheresis ${ }^{7}$. PE with immunosuppressive therapy may also be useful in cases of idiopathic rapidly progressive glomerulonephritis in children ${ }^{8}$. Instead of waiting for anti-GBM antibody and renal biopsy results, PE should be undertaken on admission. The current case with severe renal dysfunction requiring HD had a poor prognosis.

GPS is associated with a high mortality rate. Early and appropriate treatment may reverse the extent of renal damage and prevent the need for HD. Initial serum $\mathrm{Cr}$ level is a strong predictor of final renal outcome9. Patients with an initial $\mathrm{Cr}>6.6 \mathrm{mg} / \mathrm{dl}$ or who are dialysis dependent have irreversible glomerular injury and likely will not recover renal function ${ }^{6}$. Levy reported that cases presenting with dialysis-dependent renal failure had 65\% patient survival and $8 \%$ renal survival at 1 year, and 36\% patient survival and 5\% renal survival at last followup 1 year ${ }^{10}$. Advanced renal failure at presentation and/or crescents affecting more than $50 \%$ of glomeruli is a poor prognostic sign ${ }^{11}$. The diagnosis of GPS should therefore be considered in all children with rapidly progressive glomerulonephritis or pulmonary haemorrhage, and treatment should be initiated as soon as possible. This case presented with an unusually high serum $\mathrm{Cr}$ of $11.0 \mathrm{mg} / \mathrm{dl}$, consistent with extensive renal damage. She had 6 days of oliguria prior to admission. As she had a fever and we were waiting for anti-GBM antibody and renal biopsy results, methylprednisolone and $\mathrm{PE}$ were started 17 days after the onset of oliguria. Even though she was treated with PE, HD, prednisolone and cyclophosphamide, renal function did not improve.

Turner's syndrome is one of the most frequent chromosome disorders encountered in clinical practice. The most common signs are growth retardation and failure of normal sexual development at puberty. GH therapy can result in a remarkable gain in height ${ }^{12}$. This patient had showed a good response to GH therapy, with a height gain of $8 \mathrm{~cm}$ in 8 months. Unfortunately she did not receive further GH after starting HD, as her parents were unable to manage both financially. She had no further height gain after her GPS diagnosis. We have not seen any other reports of GPS associated with Turner syndrome, and the relationship between the two syndromes should be further researched.

Renal transplantation can be performed in children requiring chronic HD after disappearance of the circulating anti-GBM antibodies. There are no data indicating the recurrence rate in children after renal transplantation. Graft loss due to recurrence has been estimated to be approximately $14 \%$ in adults, but the recurrence of anti-GBM antibodies has rarely been documented ${ }^{13}$.

In conclusion, GPS is rare in children, and we present the first reported case of GPS associated with 
Turner's syndrome. Prompt diagnosis and initiation of treatment with PE, steroids and immunosuppression should be undertaken as soon as possible in GPS.

\section{References}

1. Salant DJ. Goodpasture's disease-new secrets revealed. New England Journal of Medicine 2010; 363 (4): 388-391

2. Simpson IJ, Doak PB, Williams LC, et al. Plasma exchange in Goodpasture's syndrome. American journal of nephrology 1982; 2 (6): 301-311

3. Dhruval P, Noel N, Allan T. Anti-glomerular basement membrane disease superimposed on membranous nephropathy: a case report and review of the literature. Journal of Medical Case Reports 2010; 4 (1): 237-241

4. Poddar B, Singhal S, Azim A, Gulati S, Baronia A. Goodpasture's syndrome in children. Saudi Journal of Kidney Diseases and Transplantation 2010; 21 (5): 935-939

5. Bigler SA, Parry WM, Fitzwater DS, Baliga R. An 11-month-old with anti-glomerular basement membrane disease. American journal of kidney diseases 1997; 30 (5): 710-712

6. Szczepiorkowski ZM, Winters JL, Bandarenko $\mathrm{N}$, et al. Guidelines on the use of therapeutic apheresis in clinical practice-Evidence-based approach from the apheresis applications committee of the American Society for Apheresis. Journal of clinical apheresis 2010; 25 (3):
83-177

7. Hajime N, Michiko A, Atsunori K, etal. A case report of efficiency of double filtration plasmapheresis in treatment of Goodpasture's syndrome. Therapentic Apheresis and Dialysis 2009; 13 (4): 373-377

8. Bakkaloglu SA, Kasapkara CS, Soylemezoglu O, et al. Successful management of anti-GBM disease in a 51/2-year-old girl. Nephrology Dialysis Transplantation 2006; 21 (10): 2979-2981

9. Lazor R, Bigay-Game L, Cottin V, et al. Alveolar hemorrhage in anti-basement membrane antibody disease: a series of 28 cases. Medicine 2007; 86 (3): 181-193

10. Levy JB, Turner AN, Rees AJ, Pusey CD. Longterm outcome of anti-glomerular basement membrane antibody disease treated with plasma exchange and immunosuppression. Annals of Internal Medicine 2001; 134 (11): 1033-1042

11. Salam N, Rezki H, Fadili W, Hachim K, Ramdani B. Goodpasture's syndrome-Four Case Reports. Saudi Journal of Kidney Diseases and Transplantation 2007; 18 (2): 235-238

12. Schonhoff P, Korner A, Kratzsch J, Pfaffle R, Kiess W. Long term clinical management of girls with Turner syndrome at a center of pediatric endocrinology. Experimental and clinical endocrinology \& diabetes 2011; 119 (06): 327-333

13. Cochat P, Fargue S, Mestrallet G, et al. Disease recurrence in paediatric renal transplantation. Pediatric Nephrology 2009; 24 (11): 2097-2108 